\title{
Femtosecond Laser Damage in Metals and Semiconductors During TriBeam Tomography
}

\author{
M.P. Echlin ${ }^{1}$, M. Titus ${ }^{1}$, W.C. Lenthe ${ }^{1}$, M. Straw ${ }^{2}$, P. Gumbsch ${ }^{3,4}$, and T.M. Pollock ${ }^{1}$ \\ ${ }^{1}$ Materials Department, University California at Santa Barbara, Santa Barbara, USA \\ ${ }^{2}$ FEI Company, Hillsboro, OR, USA \\ ${ }^{3}$ Fraunhofer IWM, Freiburg, Germany \\ ${ }^{4}$ Institute of Applied Materials (IAM), Karlsruhe Institute of Technology, Germany
}

The incorporation of ultrashort pulsed lasers into a dualbeam electron microscope, otherwise known as the TriBeam, has enabled applications such as the fast removal of material for tomography [1], micromachining, plasma based chemical diagnostics [2], and beam chemistry [3]. The unique low damage properties of commercial femtosecond lasers arise from the large impulse of energy imparted into the electronic structure of a material over time periods typically between 20-500 femtoseconds yielding complicated thermo-mechanical loading.

TriBeam tomography has been performed on systems ranging from polymer composites and electronic materials to metallic and ceramic structural materials [3]. Promising early results showed that laserinduced damage could be limited so that microanalytical techniques such as electron backscatter diffraction (EBSD) and energy dispersive x-ray spectroscopy (EDS) could be performed on the ablated surfaces $[1,3,4,5]$. However, simulations show that GPa-scaled shockwaves are generated during the ablation event resulting from femtosecond laser pulses with energy near the ablation-threshold of the sample material [6,7]. During such an event, compressive and tensile waves are driven into the sample subsurface, which have implications for material modification.

A number of strategies have been implemented to limit femtosecond laser damage, including the modification of the beam scanning orientation with respect to the sample surface, various beam focusing optics, and optimizing laser processing parameters. A schematic showing the typical experimental setup during femtosecond laser sectioning for tomography is shown in Figure 1. For particularly damage sensitive laser-materials operations, secondary near-glancing angle beam milling has been performed on laser ablated sample surfaces. For instance, low and high accelerating voltage gallium FIB milling and high current, low beam energy focused argon ion beams have been used to subsequently remove 10-100 $\mathrm{nm}$ depths from laser-ablated sample surfaces.

The subsurface damage has been investigated for a wide range of soft and hard materials, such as highly annealed copper $(\mathrm{Cu})$, strontium titanate (STO), polycrystalline nickel alloy (Ni-alloy), single crystal silicon ( $\mathrm{Si})$, and gallium nitride $(\mathrm{GaN})$. FIB-SEM cross-sectioning and TEM analysis was used to determine dislocation injection depths and character, phase changes, and other structural changes that were generated as a result of the laser ablation process for this wide range of materials. For example, in Figure 2 bright field TEM images collected from femtosecond laser ablated STO cross-sections of the sample surface are shown. However, the damage mechanisms are not only limited to dislocation injection, for instance in $\mathrm{Si}$ amorphization of the near subsurface region has been observed. 
Generally, light induced periodic surface structures (LIPSS) were observed for all laser-ablated samples, with LIPSS wavelengths having a fractional dependence on the $780 \mathrm{~nm}$ light used to irradiate the materials. The amplitude of the LIPSS were typically $100 \mathrm{~s}$ of $\mathrm{nm}$ in height.

\section{References:}

[1] M.P. Echlin et al, Rev. Sci. Instrum. 83 (2012) p. 023701.

[2] M.P. Echlin et al, Adv. Mater. 23 (2011) p. 2339-2342.

[3] M.P. Echlin et al, Materials Characterization: Tutorial Review 100 (2015) p. 1-12.

[4] M.P. Echlin et al, Microscopy and Microanalysis 11 (2013) p..

[5] X. Sedao et al, Applied Surface Science. 302(2014) p. 114 - 117.

[6] B.J. Demaske et al, Physical Review B. 82(2010) p. 064113.

[7] C. Wu et al, Physical Review B. 91(2015) p. 035413.

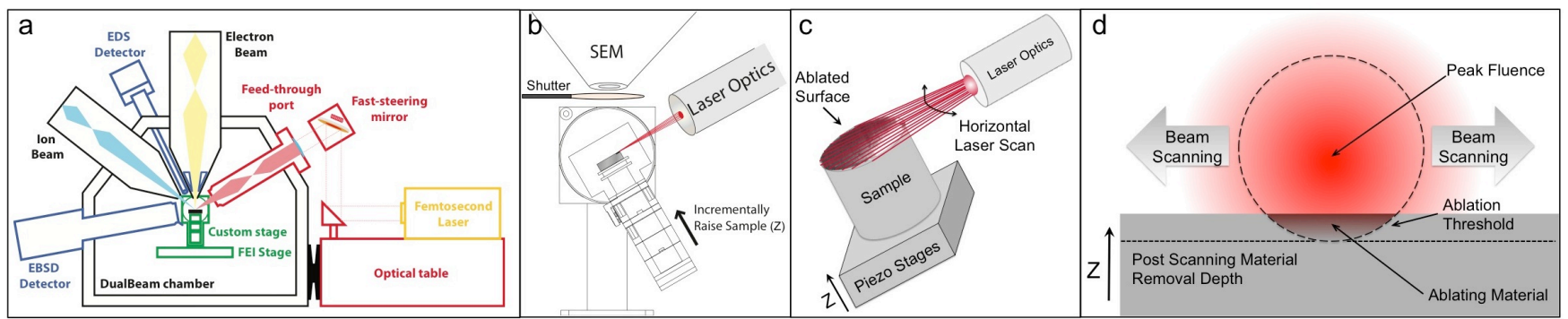

Figure 1. (a) TriBeam microscope showing laser beam feed-through port and stage location. (b) Typical surface parallel to beam laser ablation configuration. (c) Schematic of laser beam scanning horizontally, along one parallel plane to the sample surface. (d) Schematic referenced as viewed along beam propagation direction, indicating the beam scanning direction and the laser-material interaction volume.

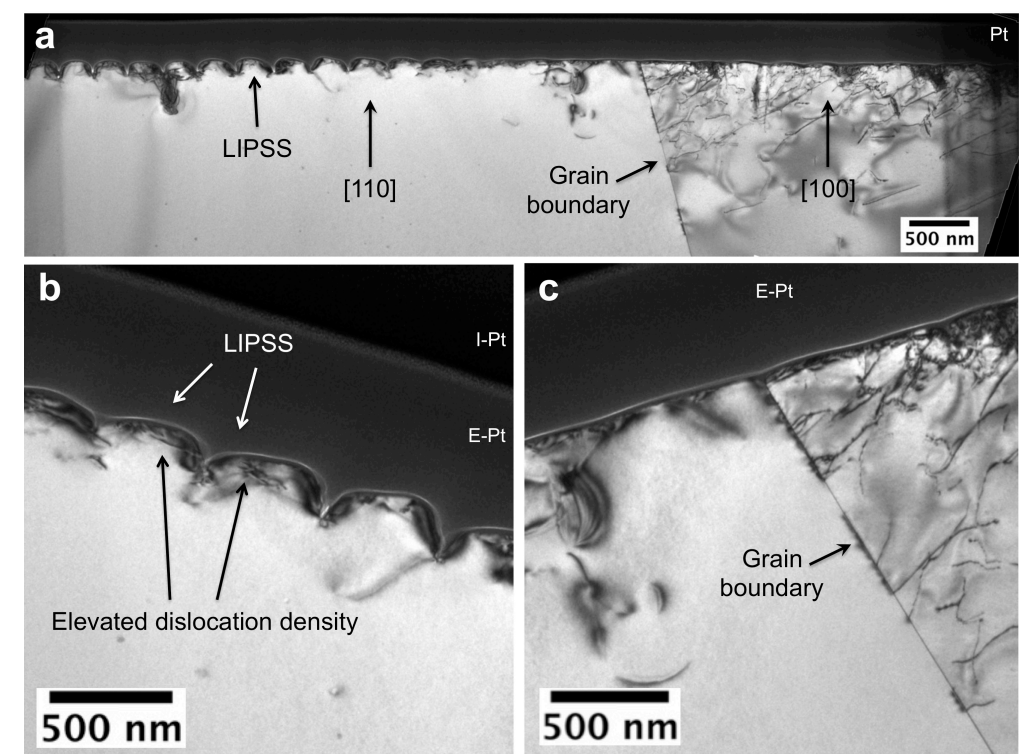

Figure 2. Bright field TEM images collected from cross-sections of femtosecond laser ablated STO surfaces. (a) Grains with [110] and [100] orientation exhibiting different dislocation densities subsurface to laser ablation. LIPSS structures are visible at the surface. (b) Detailed view showing the confinement of dislocations to the near LIPSS surface in the [110] oriented grain. (c) Detailed view of the grain boundary. 